\section{THE PHYSICIST'S CONCEPTION OF NATURE \\ Edited by JAGDISH MEHRA}

Accounts of the principal conceptions of modern physics, in historical perspective, by some of their most distinguished creators and exponents - an international symposium on "The Development of the Physicist's Conception of Nature in the Twentieth Century". held in Miramare, Trieste, Italy, 18-25 September 1972.

Contents: Space, Time and Geometry: Contributions by D. W. Sciama, S. Chandrasekhar, P. A. M. Dirac, P. Jordan, J. Ehlers, J. Mehra, A. Trautman J. A. Wheeler. - Quantum Theory: Contributions by L. Rosenfeld, W. Heisenberg, B. L. van der Waerden, P. Jordan, J. M. Jauch, E. P. Wigner, F. Rohrlich, R. E. Peierls, G. Wentzel, S. Tomonaga J. Schwinger, A. Salam, C. N Yang, V. G. Telegdi, H. B. G. Casimir. - Statistical Description of Nature: Contributions by G. E. Uhlenbeck, M. Kac, W. E. Lamb Jr., E. G. D. Cohen, I. Prigogine M. Eigen. - Physical Description. Epistemology and Philosophy Contributions by C. F. von Weizsäcker, L. N. Cooper, J. M. Jauch, J. S. Bell, R. Haag, I. Prigogine, G. Ludwig, D. Finkelstein, B. d'Espagnat, C. F. von Weizsäcker.

Cloth, approx. 820 pages

Price: Dfl. 200,-/US\$ 75.00 (approx.)

"This symposium brought back the past and put the great achievements of physics in clearer perspective than any conference I have ever attended. What a marvellous collection of speakers and topics!"

John Archibald Wheeler (Princeton University)

D. REIDEL PUBLISHING COMPANY

Dordrecht-Holland/

Boston-U.S.A.

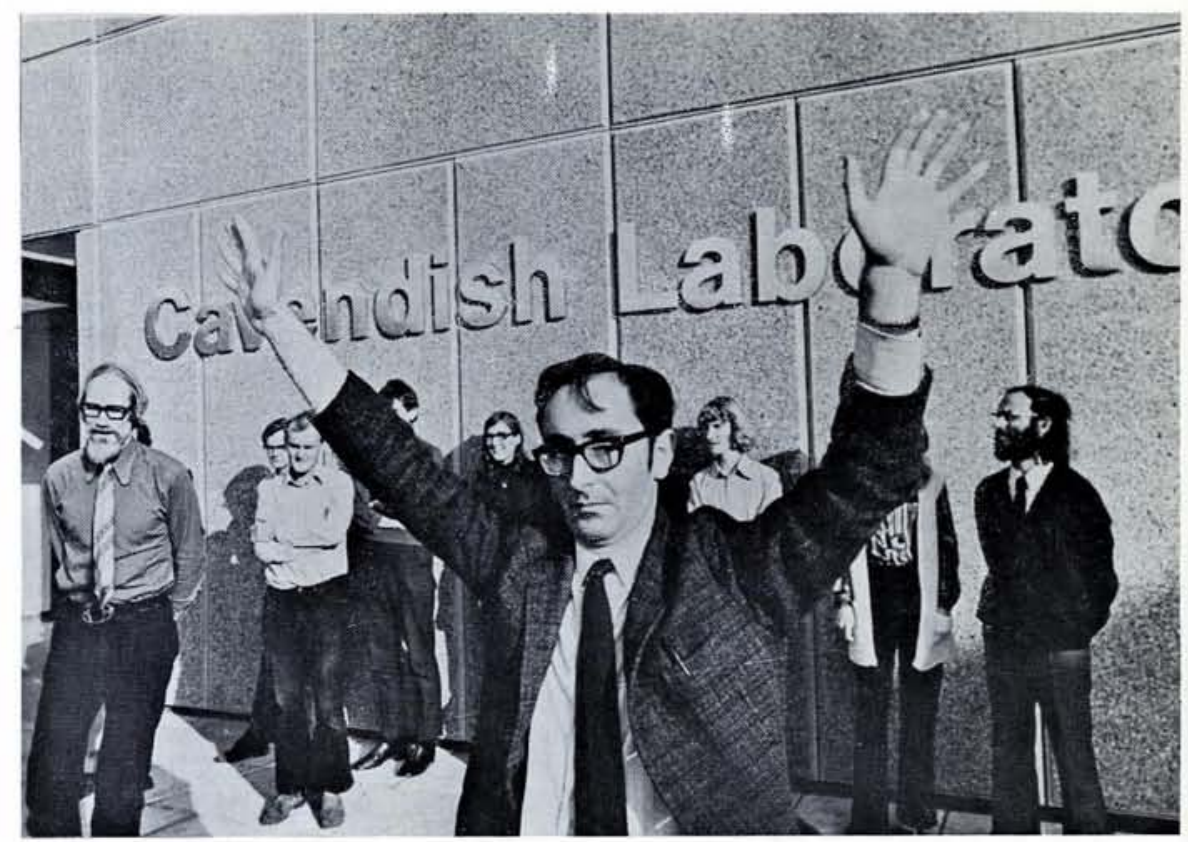

B.D. Josephson celebrates his Nobel Prize award at the University of Cambridge

copic quantum effect. At finite voltages, $V$, electromagnetic radiation will be generated according to the relation $\mathrm{hv}=2 \mathrm{eV}$. The proportionality factor $\frac{2 \mathrm{e}}{\mathrm{h}} \sim 500 \mathrm{MHz} \mu \mathrm{V}^{-1}$

contains only fundamental constants.
Based on the above relation it was possible to determine $\frac{h}{e}$ with high accuracy and to create a new voltage standard by reducing a voltage measurement to a more simple frequency measurement.

\title{
The Physics of the Rare Earth Metals
}

\author{
Elsinore, Denmark, 29 August - 1 September 1973
}

A Europhysics Study Conference on the Rare Earth Metals was held at the LO-Højskole, Elsinore, Denmark, in the period 29 August-1 September, 1973, immediately following the International Magnetism Conference in Moscow. The conference was sponsored by the Metals and Magnetism Sections of EPS and by the Danish Atomic Energy Commission. The National Science Foundation provided partial travel support for some participants from the U.S.A.

The LO-Højskole is an educational centre belonging to the Danish Trades Unions, and it is situated by the sea, in very pleasant surroundings, about 25 miles from Copenhagen. In order to restrict the number of participants to approximately 100 , it was necessary to reject a number of applications to attend the conference. It was a truly international meeting, with participants from 16 different countries. The summary session was held at the Danish AEC Research Establishment Risø, after which the participants had an opportunity of seeing the establishment, especially the neutron scattering facilities.

The philosophy and organization of the conference followed broadly that of the Gordon Conferences, with emphasis on informal discussions, and no published proceedings. The aim was to review the present experimental and theoretical understanding of the physics of the rare earth metals, and this was accomplished principally by means of invited half-hour review papers and the resulting discussions, led by the session chairman. There was also the opportunity to present and discuss short contri- 
butions, however, and 30 such fiveminute contributed papers were given. The programme was arranged by an International Advisory Committee consisting of

R.J. Elliott (Oxford University) ;

W.C. Koehler (Oak Ridge National Laboratory) ;

S. Legvold (lowa State University) ;

A.R. Mackintosh (Danish AEC, Risø), Chairman ;

H. Bjerrum Møller (Danish AEC, Risø).

The programme of invited papers was as follows (name of chairman in parentheses after title of session).

ELECTRONIC STRUCTURES (T. Nagamiya)

The Electronic Structures of the Rare Earth Metals and Actinides

A.J. Freeman (Northwestern)

Photoemission Studies of Rare Earth Metals Y. Baer (ETH)

Renormalized Atom Theory of Rare Earth Metals R.E. Watson (Brookhaven

MAGNETIC STRUCTURES AND MAGNETIZATION (W.C. Koehler)

Magnetic Structures and Conduction Electron Spin Densities

R.M. Moon (Oak Ridge)

Magnetic Form Factors in Rare Earth Metals and Compounds

T.O. Brun (Argonne)

High Field Magnetization of Rare Earth Metals L.W. Roeland (Amsterdam)

\section{VALENCE CHANGES (S.H. Liu)}

Theory of Valence Transitions

L.M. Falicov (Berkeley)

The Electronic Properties of $\mathrm{Ce}$

B. Coqblin (Orsay)

Demagnetization of Rare Earth Ions during

Valence Changes

D. Wohlleben (La Jolla)

\section{MAGNONS AND PHONONS (S. Legvold)}

Magnon Dispersion Relations and Exchange in Rare Earth Metals

R.M. Nicklow (Oak Ridge)

Magnetoelastic Effects and Elastic Constants J. Jensen (Riso)

Phonons, Magnon-Phonon Interactions

and Magnetic Anisotropy

J.C.G. Houmann (Risø)

SINGLET GROUND STATES AND HYPERFINE INTERACTIONS (B.R. Cooper)

Magnetic Properties of $\mathbf{P r}$

B.D. Rainford (Imperial)

Singlet Ground 'States in Rare Earth Compounds R.J. Birgeneau (Bell)

Rare Earth Hyperfine Interactions

R.L. Cohen (Bell)

DILUTE ALLOYS AND DOMAINS (J.J. Rhyne) Electron Spin Resonance in Dilute Rare Earth Alloys

R. Orbach (UCLA)

Superconductivity and Electron Scattering

in Dilute La Alloys

D.K. Finnemore (Ames)

Magnetic Domains in Rare Earth Metals

T. Egami (Stuttgart)
SUMMARY AND DISCUSSION (H. Bjerrum Moller)

Theoretical

R.J. Elliott (Oxford)

Experimental

A.R. Mackintosh (Riso)

As a result of these lectures, the short communications and the extensive discussions which took place, our present understanding of the rare earth metals was reviewed and placed in perspective, while promising lines for future research were outlined. Our experimental understanding of the magnetic properties of the heavy rare earths has reached a very sophisticated level, so that subtle effects of, for example, anisotropic exchange interactions may be studied in great detail. On the other hand, the theoretical understanding of the magnetic interactions is less satisfactory and further theoretical and experimental studies of the origins of the crystalline electric fields are now required. These crystal fields are of particular importance in the light rare earths, which have been relatively little studied experimentally, although a fairly thorough understanding of Praseodymium is beginning to emerge. The valence changes which occur in Cerium and a number of rare earth compounds, due to the partial promotion of a $4 \mathrm{f}$ electron to the conduction band, give rise to a fascinating variety of physical phenomena which are not yet understood in detail. During recent years a large amount of systematic work has been performed on well-characterized rare earth compounds, in which the environment of the rare earth ion has a decisive effect on the magnetic properties. A striking example of such behaviour is provided by ions whose crystal field ground-state is a nonmagnetic singlet. Both the experimental and theoretical understanding of such systems contain obscurities which require further examination. In general, it was felt that the complex magnetic interactions to which rare earth ions may be subjected, and the resulting rich variety of magnetic properties, are most likely to be further elucidated in the future. Measurements on single crystals of compounds and on dilute alloys will use the extensive selection of experimental techniques, such as magnetization and transport measurements, electron spin- and nuclear-resonance, photoemission and especially neutron diffraction, which were discussed at the conference.

\section{A.R. Mackintosh}

J.G. Houmann
New Books from North-Holland

\section{Crystal Growth An Introduction}

edited by P. HARTMAN, Rijksuniversiteit, Leiden, The Netherlands

(North-Holland Series in Crystal Growth, $\mathrm{Vol}, 1)$

1973. 544 pages.

Dfl. 60.00 (about US $\$ 24.00$ )

The book is primarily intended for anyone entering the field of crystal growth research or for those who have to deal with crystal growth problems in various branches of science, such as solid state
physics and chemistry, materials science, physics and chemistry, materials science, ing, crystallography and mineralogy.

CONTENTS: Nucleation and Epitaxy. Techniques of Crystal Growth. Theory of Crystal Growth. The Properties and Observation of Dislocations.

\section{Computed Electron Micrographs and Defect Identification}

by A.K. HEAD, P. HUMBLE, L.M. CLAREBROUGH, A.J. MORTON and C.T. FORWOOD, CSIRO-Division of Tribophysics, University of Melbourne, Australia

(Defects in Crystalline Solids, Vol. 7).

1973. 410 pages.

Dfl. 100.00 (about US $\$ 40.00$ ).

The book is intended for the postgraduate student or research worker using transmission electron microscopy for studying defects in crystalline solids.

CONTENTS: Introduction to the basic theory. Experimental techniques. Principles of ONEDIS. Matching with ONEDIS. Principles of TWODIS. Matching with TWODIS. Application of the technique. Discussion of the applications and limitations of the technique. Computer programs.

\section{Quantum Mechanics New Approaches to Selected Topics}

by H.J. LIPKIN, Weizmann Institute of Science, Rehovoth, Israel.

1973. 480 pages.
Dfl. 95.00 (about US $\$ 38.00$ ).

CONTENTS: Polarized photons and quantum theory. The Mössbauer effect. The Mössbauer effect in a solid. The Mössbauer effect and momentum transfer to bound systems. Identical particles and second quantization. Identical composite particles and bound systems. Kaon decay. One-dimensional scattering in quantum mechanics. The many-body problem in quantum mechanics. Pairing correlation tions in many-body systems. Fexnman dins in dugrams, propagators and fields. Thtronics Invariance symmetry transformations and conservation laws. The Lorentz group.

NORTH $\mathrm{NH}$

P.O. Box 211, Amsterdam, The Netherlands Distributed in the United States and Canada by American Elsevier Publishing Company, Inc., 52 Vanderbilt Avenue, New York, N.Y. 10017. 